\title{
A Hilbert-Huang Transform-Based Adaptive Fault Detection and Classification Method for Microgrids
}

\author{
Yijin Li ${ }^{1}$, Jianhua $\operatorname{Lin}^{1}$, Geng Niu ${ }^{2, *}$, Ming $W^{2}{ }^{2}$ and Xuteng Wei ${ }^{1}$ \\ 1 School of Mechanical Electronic and Information Engineering, China University of Mining and \\ Technology-Beijing, Haidian District, Beijing 100083, China; yijinli@cumtb.edu.cn (Y.L.); \\ 18801083527@163.com (J.L.); xuteng_wei@163.com (X.W.) \\ 2 State Grid Shanghai Energy Interconnection Research Institute, China Electric Power Research Institute, \\ Haidian District, Beijing 100192, China; wuming@epri.sgcc.com.cn \\ * Correspondence: niugeng@epri.sgcc.com.cn; Tel.: +86-010-8281-4828 (ext. 1001)
}

check for updates

Citation: Li, Y.; Lin, J.; Niu, G.; Wu, M.; Wei, X. A Hilbert-Huang Transform-Based Adaptive Fault Detection and Classification Method for Microgrids. Energies 2021, 14, 5040 https://doi.org/10.3390/en14165040

Academic Editors: Adel Merabet and J. C. Hernandez

Received: 29 July 2021

Accepted: 12 August 2021

Published: 17 August 2021

Publisher's Note: MDPI stays neutral with regard to jurisdictional claims in published maps and institutional affiliations.

Copyright: (c) 2021 by the authors. Licensee MDPI, Basel, Switzerland. This article is an open access article distributed under the terms and conditions of the Creative Commons Attribution (CC BY) license (https:/ / creativecommons.org/licenses/by/ $4.0 /)$.

\begin{abstract}
Fault detection in microgrids is of great significance for power systems' safety and stability. Due to the high penetration of distributed generations, fault characteristics become different from those of traditional fault detection. Thus, we propose a new fault detection and classification method for microgrids. Only current information is needed for the method. Hilbert-Huang Transform and sliding window strategy are used in fault characteristic extraction. The instantaneous phase difference of current high-frequency component is obtained as the fault characteristic. A self-adaptive threshold is set to increase the detection sensitivity. A fault can be detected by comparing the fault characteristic and the threshold. Furthermore, the fault type is identified by the utilization of zero-sequence current. Simulations for both section and system have been completed. The instantaneous phase difference of the current high-frequency component is an effective fault characteristic for detecting ten kinds of faults. Using the proposed method, the maximum fault detection time is $13.8 \mathrm{~ms}$ and the maximum fault type identification time is $14.8 \mathrm{~ms}$. No misjudgement happens under non-fault disturbance conditions. The simulations indicate that the proposed method can achieve fault detection and classification rapidly, accurately, and reliably.
\end{abstract}

Keywords: fault detection; instantaneous phase difference of current high-frequency component (IPDCHC); Hilbert-Huang Transform (HHT); self-adaptive threshold; microgrid

\section{Introduction}

A microgrid is a small power supply system which contains distributed generations (DGs), loads, energy storage devices, monitoring, protection, and automation equipment [1]. It has several advantages, such as high power efficiency and power supply reliability, flexible operation and control mode, and low carbon emissions, which are beneficial to the power system and society [2-4].

With the high-proportional and multi-typed integration of DGs into microgrids [5], fault points in microgrids may be located very close to power sources. These faults may cause customer interruptions, trips of DGs, and unstable voltage frequency, which have a negative influence on system safety and stability [6]. Thus, faults should be rapidly and accurately detected and cleared from a microgrid.

Fault detection, which is the key to the protection of a microgrid, plays an important role in microgrid technique [7]. It can quickly determine the faults and facilitate fast fault isolation and power service restoration. However, fault detection in microgrids is largely different from traditional fault detection. Due to the penetration of DGs, power flow becomes bidirectional. The fault currents can inject to the fault point from both the upstream side and the downstream side [8]. The level of fault current provided by DG varies according to the type of DG, power capacity, distance to the fault point, etc. [9]. Moreover, owing to the flexible operation modes and response to consumers, the operating 
status of microgrids is more complicated [10-12]. These features make fault characteristics in microgrids more complex. Thus, rapid and accurate fault detection is urgently needed. Related research has attracted a lot of attention in recent years [13-17].

Fault detection methods can be mainly classified into time-domain methods and frequency-domain methods. Time-domain fault detection methods make the fault judgment by collecting and analyzing voltage/current signals. To reduce the impact of DG integration on distribution network protection, an algorithm based on voltage only was proposed in [18]. Through load flow and sensitivity calculations, the protection function was achieved. By comparing the calculated result with the threshold, faults can be detected and located. The authors of [19] used the positive sequence currents amplitude at the upstream and downstream of the fault point to locate the fault section. In this method, state observer based on the system model must be built. Time-domain fault detection methods are usually simple and rapid. However, the selection of threshold is difficult. The selection of an inappropriate threshold may cause a fault to be misjudged or not detected. Frequency-domain fault detection methods are achieved by the transformation of time-domain signal to frequency-domain signal and data analysis. The authors of [20] proposed a new algorithm for busbar protection based on Hilbert-Huang Transform. The instantaneous amplitude of a travelling wave is extracted as the fault feature. Though frequency-domain methods hold a higher accuracy and sensitivity, the calculations are more complicated compared with time-domain methods. Moreover, the anti-interference capacity and self-adaptivity should be improved in these methods. Machine learning provides another method for fault detection [3]. The theory of this kind of method is simple, and the method is easy to carry out with a high accuracy. However, the calculation is complex. When the model parameters change, calculations should be completed again, leading to a limited self-adaptive capacity.

After fault detection, fault classification is an important procedure to achieve splitphase trip and reduce a fault's effect on consumers in a microgrid $[8,9]$. This is one of the developing trends in smart microgrids [21]. In [22], two classifiers based on voltage angle and magnitude are proposed to determine the fault type. Only local information is needed for computation. They are applicable both for microgrids with photovoltaic DGs and any three-phase system. In [23], a new approach that uses a combination of wavelet functions and machine learning for fault classification in microgrids is introduced. Particle swarm optimization is applied to identify the optimal wavelet functions combination to extract the most prominent features. Four different classification techniques are used to automate the procedure of fault classification in microgrids. In [24], an intelligent data mining-based fault detection and classification strategy for microgrids is proposed. The sensitive fault features are extracted by preprocessing the faulted voltage and current signals through Hilbert Transform. Fault classification is completed by training the AdaBoost classifier.

Based on the above analysis, accuracy and self-adaptivity are the key problems for both the fault detection and fault classification of microgrids. In this paper, a frequencydomain method is modified and improved for accurate fault detection. A self-adaptive threshold setting method is studied to increase the self-adaptivity. Thus, a novel fault detection and classification method is proposed. The instantaneous phase difference of current high-frequency component (IPDCHC) is extracted as the fault characteristic. Hilbert-Huang Transform (HHT) and the sliding window strategy are used during the calculation. A self-adaptive threshold setting method is provided. On the basis of IPDCHC and the self-adaptive threshold, a fault detection method is proposed. Taking zero-sequence current into consideration, a fault classification method is further proposed. Simulations for both section and system are completed with MATLAB/Simulink to test the validity of the proposed method.

The remainder of the paper is composed of four sections as follows. The extraction method and analysis of the proposed fault characteristic is described in Section 2. The fault detection method and fault classification method are illustrated in Section 3. Simulation results are provided and discussed in Section 4. Finally, the paper is concluded in Section 5. 


\section{Fault Characteristic Extraction Method and Analysis}

Fault characteristic is the key to the fault detection of microgrids. A fault characteristic with a high sensitivity leads to rapid fault detection. Hilbert-Huang Transform is an effective transform method for signal analysis. Thus, to achieve fast fault detection in microgrids, a new fault characteristic extraction method is proposed based on HHT.

\subsection{Hilbert-Huang Transform}

HHT was firstly proposed by N. E. Huang et al. [25] in 1998. Developed on the basis of Hilbert Transform, HHT can deal with both non-linear signals and non-stationary signals. Moreover, it can also complete high-precision analysis in both time domain and frequency domain. Owing to HHT's good performance, it has been widely used in low-frequency vibration signal analysis, power quality analysis, mechanical structure fault diagnosis, and other fields $[26,27]$.

HHT is composed of two parts: Empirical Mode Decomposition(EMD), and Hilbert Transform.

\subsubsection{Empirical Mode Decomposition}

The EMD can decompose any signal into a finite set of Intrinsic Mode Functions (IMFs). The IMF can give a reasonable explanation for the instantaneous frequency of the signal. An IMF should satisfy the following two conditions: (1) The difference between the number of extreme points and the number of zero crossing points is no more than one. (2) The envelope lines of the local maximum and local minimum are symmetric about the time axis at any point.

The procedures of EMD are illustrated as follows.

STEP 1: Let $k=1$.

STEP 2: Obtain the maximum envelope line $b_{\max }(t)$ and minimum envelope line $b_{\min }(t)$ by fitting the maximum and minimum values of the signal $s(t)$ with the cubic spline curve.

STEP 3: Calculate the average value of the maximum envelope and minimum envelope and obtain the mean envelope $m_{k}(t)$ :

$$
m_{k}(t)=\frac{b_{\max }(t)+b_{\min }(t)}{2} .
$$

STEP 4: Obtain the $k$ th component $h_{k}(t)$ using the following formula:

$$
h_{k}(t)=s(t)-m_{k}(t) .
$$

STEP 5: Judge whether the $h_{k}(t)$ meets the two abovementioned IMF conditions. If $h_{k}(t)$ meets the IMF conditions, let $h_{k}(t)$ be the $k$ th IMF component $i_{k}(t)$ and enter STEP 6 ; otherwise, let $s(t)=h_{k}(t)$ and return to STEP 2 .

STEP 6: Define the $k$ th residual signal as $r_{k}(t)$, then:

$$
r_{k}(t)=s(t)-i_{k}(t)
$$

Judge whether the $r_{k}(t)$ meets the terminal condition. If $r_{k}(t)$ meets the terminal condition, the EMD process ends; otherwise, let $s(t)=r_{k}(t), k=k+1$, and return to STEP 2 .

The terminal condition mentioned above can be any one among the following three conditions:

(1) The $r(t)$ is a constant;

(2) The $r(t)$ is a monotonic function;

(3) The $r(t)$ has only one extreme point.

Through EMD, IMF1 is the most high-frequency component of all the IMFs [28]. Thus, in this paper, IMF1 is used for the following Hilbert Transform. 


\subsubsection{Hilbert Transform}

A time series signal $X(t)$ can be transformed to signal $Y(t)$ by Hilbert Transform as follows:

$$
Y(t)=\frac{1}{\pi} P \int_{-\infty}^{+\infty} \frac{X(\xi)}{t-\xi} d \xi
$$

In Equation (4), $P$ is the Cauchy principal value.

When the two signals $X(t)$ and $Y(t)$ form complex conjugate, an analytic function $Z(t)$ can be constructed as:

$$
Z(t)=X(t)-j \cdot Y(t)=a(t) e^{j \theta(t)} .
$$

In Equation (5), $a(t)$ is the instantaneous amplitude of $Z(t)$, and $\theta(t)$ is the instantaneous phase of $Z(t)$. There are:

$$
\begin{gathered}
a(t)=\sqrt{X^{2}(t)+Y^{2}(t),} \\
\theta(t)=\arctan \frac{Y(t)}{X(t)} .
\end{gathered}
$$

The instantaneous frequency $f(t)$ can be further obtained through the following equation:

$$
f(t)=\frac{1}{2 \pi} \frac{d \theta(t)}{d t} .
$$

Based on the instantaneous amplitude and instantaneous phase, the time-frequency domain analysis of the signal can be carried out.

\subsubsection{Sliding Window Strategy}

During fault characteristic extraction, real-time features should be ensured. However, in HHT EMD requires a certain data length to ensure a valid IMF output. This leads to the problems of time-consuming for fault characteristic extraction method using HHT. To reduce the influence caused by data preparation, the sliding window strategy is used in the proposed method. With this strategy, a sample with fixed length can be obtained by the sliding of a fixed-length window under a fixed interval of time [29]. The sample is updated through sliding along with time. After a fixed interval of time, some new data come in and the old data of the same quantity in the rear are abandoned. An updated sample with a fixed length is formed. In this way, the data for EMD can be prepared rapidly. Moreover, the proper selection of sliding window parameters will further improve the strategy's performance.

\subsection{Extraction and Analysis of the Fault Characteristics}

A new fault characteristic based on HHT is proposed in this study. The instantaneous phase of the current high-frequency component is calculated. By subtraction, the difference between the instantaneous phases can be used as the fault characteristic for rapid fault detection. The extraction process of the proposed fault characteristic is shown in Figure 1. 


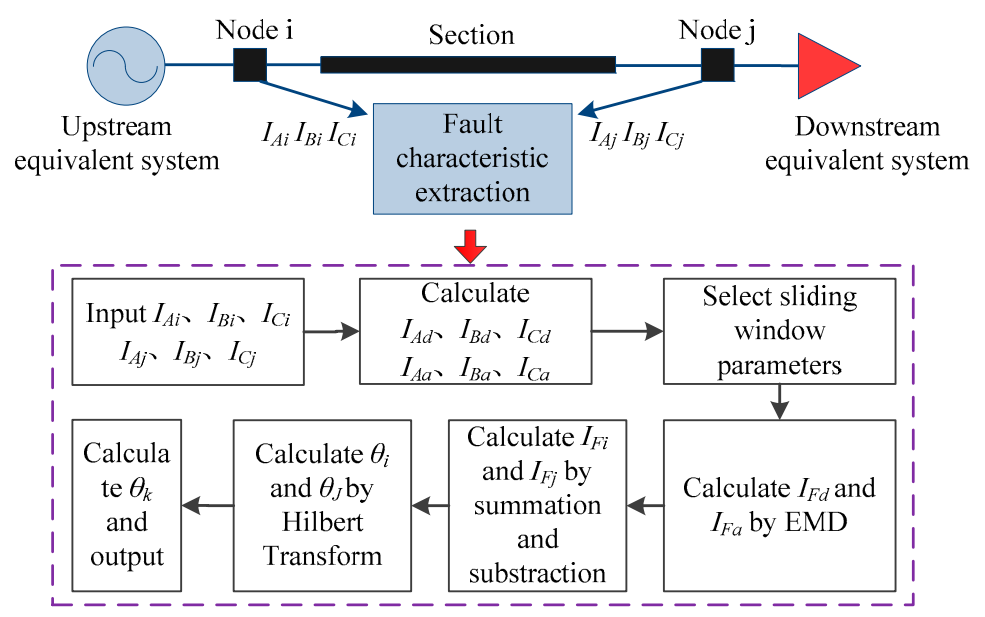

Figure 1. Extraction process of the proposed fault characteristic.

Firstly, for a section, the three-phase currents of upstream node $\left(I_{A i}, I_{B i}, I_{C i}\right)$ and downstream node $\left(I_{A j}, I_{B j}, I_{C j}\right)$ are collected. The certain phase currents' difference $\left(I_{d}\left(I_{A d}\right.\right.$, $\left.\left.I_{B d}, I_{C d}\right)\right)$ and the certain phase currents' sum $\left(I_{a}\left(I_{A a}, I_{B a}, I_{C a}\right)\right)$ can be obtained by vector calculations. Taking phase A as an example, $I_{A d}$ and $I_{A a}$ can be calculated by the following equations:

$$
\left\{\begin{array}{l}
I_{A d}=I_{A i}-I_{A j} \\
I_{A a}=I_{A i}+I_{A j}
\end{array} .\right.
$$

Secondly, a sliding window of each phase currents' difference with a fixed length is selected. In this paper, 200 sample points are used to build a sliding window. EMD is carried out with the sliding window data. The current difference's high-frequency component of each phase $\left(I_{F d}\left(I_{F A d}, I_{F B d}, I_{F C d}\right)\right)$ is extracted, which is the IMF1. The current sum's high-frequency component of each phase $\left(I_{F a}\left(I_{F A a}, I_{F B a}, I_{F C a}\right)\right)$ is extracted in the same way. $I_{F i}$ and $I_{F j}$ are defined as the node current's high-frequency component, which can be calculated by the following equations:

$$
\left\{\begin{array}{l}
I_{F i}=\frac{I_{F a}(N)+I_{F d}(N)}{2} \\
I_{F j}=\frac{I_{F a}(N)-I_{F d}(N)}{2}
\end{array} .\right.
$$

Finally, Hilbert Transform is carried out for each phase's $I_{F i}$ and $I_{F j}$. The instantaneous phase of upstream node current's high-frequency component and that of downstream node current's high-frequency component are obtained, which are defined as $\theta_{i}$ and $\theta_{j}$. After subtraction of the two instantaneous phases, the mean value of the subtractions' absolute values is defined as the instantaneous phase difference of current high-frequency component (IPDCHC) $\theta_{k}$. The $\theta_{k}$ is extracted as the fault characteristic.

Fault simulation analysis has been completed for a microgrid with MATLAB/Simulink. The simulation model and the related parameters are shown in Figure 2. Both sections of double-ended power supply type and sections of power-load type have been taken into consideration. Figure $2 \mathrm{a}$ is used as an example for fault characteristic analysis. The sampling frequency of the signal is $5 \mathrm{kHz}$. A metallic ground fault is set $0.4 \mathrm{~km}$ away from the upstream node. The fault occurs at $0.8 \mathrm{~s}$. The waveform of the obtained IPDCHC is shown in Figure 3. 


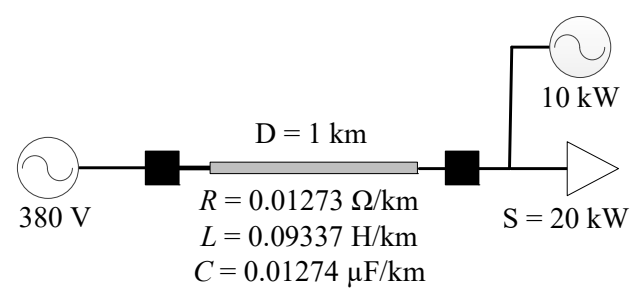

(a) Double-ended power supply type.

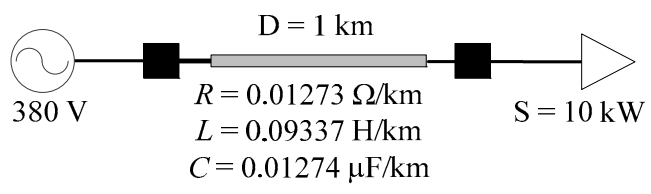

(b) Power-load type.

Figure 2. Section model for simulation.
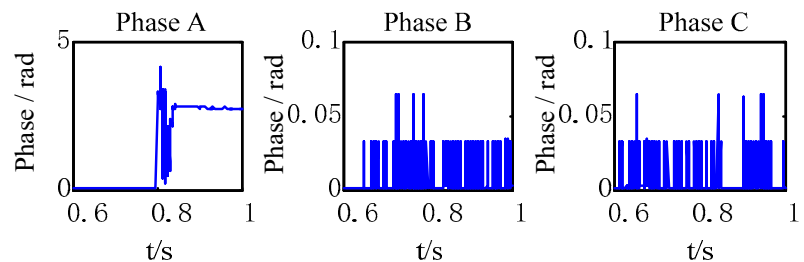

(a) A phase-to-ground fault.
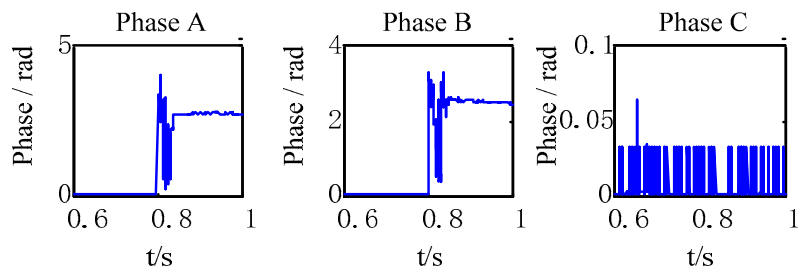

(b) A-B phase-to-ground fault.
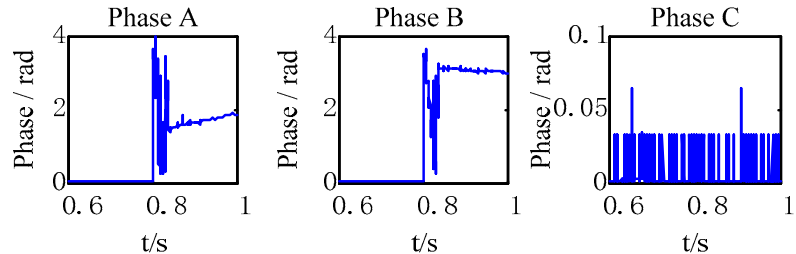

(c) A-B phase-to-phase fault
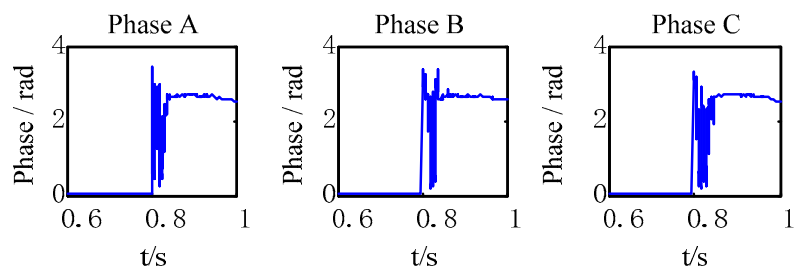

(d) Three-phase fault.

Figure 3. Typical fault simulation results.

As can be seen from Figure 3, the IPDCHC of the fault phase displays an obvious change after the fault occurrence. The IPDCHC maintains a low level (no more than $0.1 \mathrm{rad}$ ) when there is no fault. However, when a fault occurs the IPDCHC increases remarkably 
and remains at around $3 \mathrm{rad}$. The simulation results of a single phase-to-ground fault (a), a two phase-to-ground fault (b), a phase-to-phase fault (c), and a three-phase fault (d) are shown in Figure 3. The conclusions are consistent for all the situations. IPDCHC can distinguish the fault from normal situations, illustrating that IPDCHC can be used as a fault characteristic.

\section{Rapid and Self-Adaptive Fault Detection and Classification Method of Microgrid}

IPDCHC has been verified as an effective fault characteristic. Thus, according to the change feature of IPDCHC, the rapid fault detection of microgrids can be achieved. Furthermore, by measuring the zero-sequence current, the fault type can be identified online.

\subsection{Method Framework}

The proposed method contains two parts: a fault detection method and fault classification method. The overall flow chart of the method is shown in Figure 4. Firstly, node current data are collected. Then, the node data undergo a series of pre-processing procedures, including inputting the three-phase currents, calculating the nodes' phase currents' difference and phase currents' sum, and selecting the parameters of the sliding window. With the pre-processed data, HHT is carried out to extract the IPDCHC. The self-adaptive threshold of IPDCHC is calculated. The effective value and the threshold of zero-sequence current are acquired. By comparing the IPDCHC and its self-adaptive threshold, fault detection can be achieved. By comparing the effective value and the threshold of zero-sequence current, fault type can be identified. Finally, the results are output.

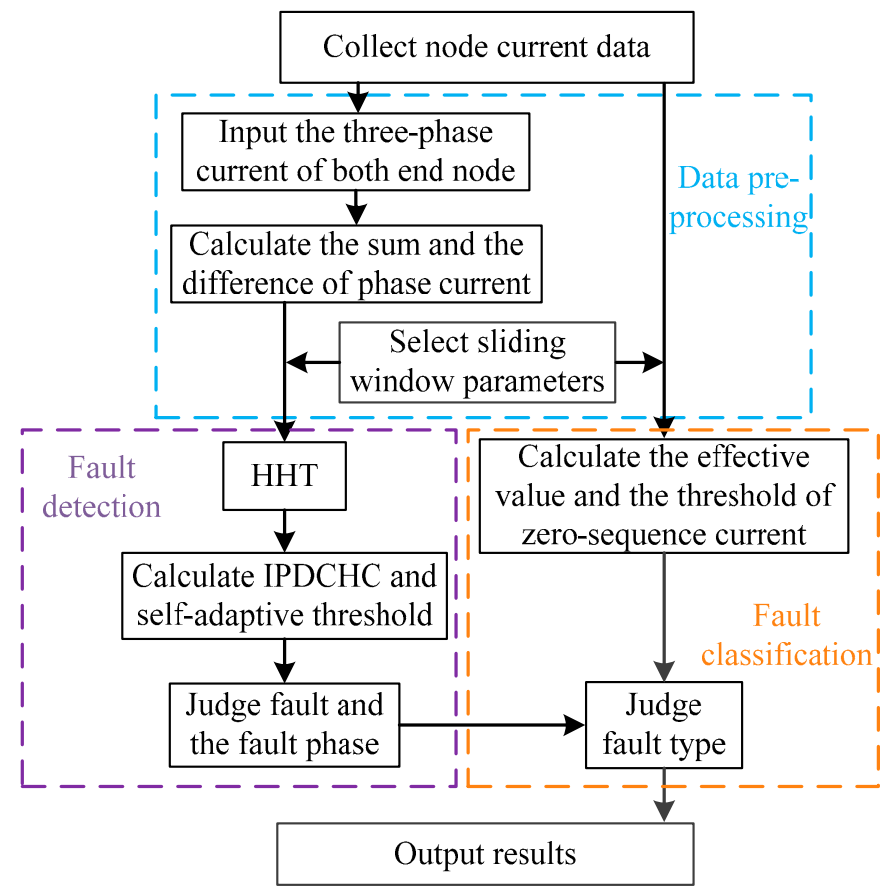

Figure 4. Overall flow chart.

\subsection{Rapid and Self-Adaptive Fault Detection Method}

\subsubsection{Procedures of the Fault Detection Method}

Fault detection is achieved by the comparing of IPDCHC and the self-adaptive threshold. The flow chart of the fault detection method is shown in Figure 5. The $\theta_{A}, \theta_{B}$ and $\theta_{C}$ represent the IPDCHC of phase A, phase $B$, and phase $C$, respectively. The $\psi_{A}, \psi_{B}$ and $\psi_{C}$ represent the threshold of each phase at the present moment. 


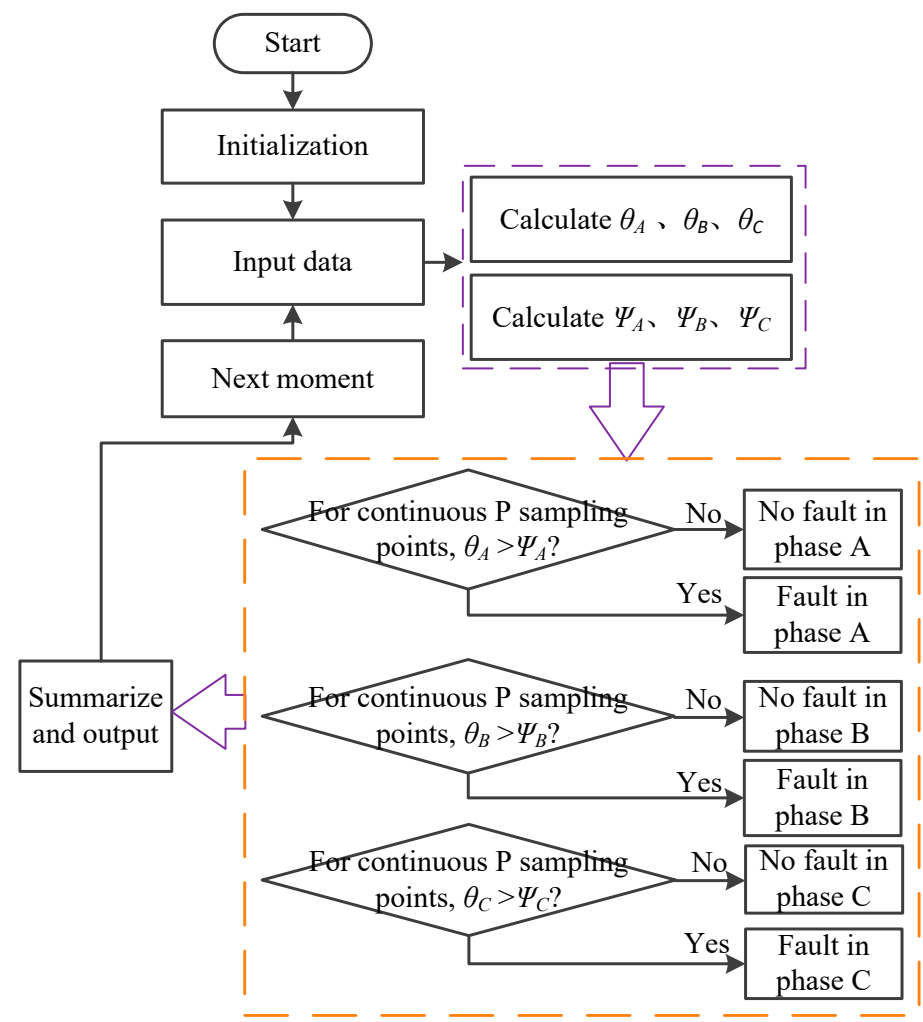

Figure 5. Flow chart of fault detection.

The specific procedures of the fault detection method are illustrated as follows:

(1) Initialization. Initialize $P, \theta_{A}, \theta_{B}, \theta_{C}, \psi_{A}, \psi_{B}$, and $\psi_{C}$.

(2) Input data of the present moment. Input the node three-phase currents and the sliding window parameters.

(3) Calculation. Calculate the IPDCHC and the threshold of each phase.

(4) Judgement. Compare the IPDCHC with the threshold for each phase. When the IPDCHC exceeds the threshold for continuous $\mathrm{P}$ sampling points, judge that the fault happens in the corresponding phase. Otherwise, no fault happens in the corresponding phase.

(5) Output. Summarize and output the fault detection result of the present moment.

(6) Turn to the next moment and return to step (2).

\subsubsection{Setting of Threshold}

To achieve rapid, accurate, and reliable fault detection, a real-time setting method of the threshold value is studied. This method makes the threshold self-adaptive. When there is no fault, the threshold value is high enough to avoid misjudgment. When a fault happens, the threshold value decreases sharply. Faults can be detected rapidly by comparing the threshold and the IPDCHC. The threshold's real-time setting equation is as follows:

$$
\Psi=\eta_{S}\left|\arctan \left(\frac{I_{a}}{G I_{d}}\right)\right| .
$$

Therein, $\psi$ is the threshold; $\eta_{s}$ is the security coefficient, generally set as $2 ; I_{a}$ is the instantaneous amplitude of certain phase current sum's high-frequency component; $I_{d}$ is the instantaneous amplitude of certain phase current difference's high-frequency component; $G$ is the sensitivity factor. $G$ will affect the change extent of $\psi$ and the detection method's anti-interference capacity. The bigger $G$ is, the more obviously $\psi$ decreases after a fault, which may lead to the misjudgement of disturbance as fault. $G$ is generally set as 100 .

The calculation procedures of threshold are described as follows:

(1) Input the node currents. 
(2) Calculation. Based on HHT, the instantaneous amplitude of certain phase current sum's/difference's high-frequency component is obtained.

(3) Using Equation (11), the threshold of IPDCHC is acquired and output.

(4) Turn to the next moment. Repeat the steps (1)-(3) to obtain the threshold of each sampling point.

\subsection{Fault Classification Method}

With the fault detection result, the next step is fault classification. In the proposed fault detection method, the fault phase is easy to identify according to IPDCHC. However, the difficulty lies in the differentiating of two phase-to-ground fault and phase-to-phase short circuit fault. The effective value of zero-sequence current is used in the proposed method. The ground fault and the short circuit fault can be distinguished by comparing the effective value of zero-sequence current and the threshold. The flow chart of fault classification is shown in Figure 6. $I_{0 i}$ and $I_{0 j}$ are the effective values of zero-sequence currents of the upstream node and downstream node.

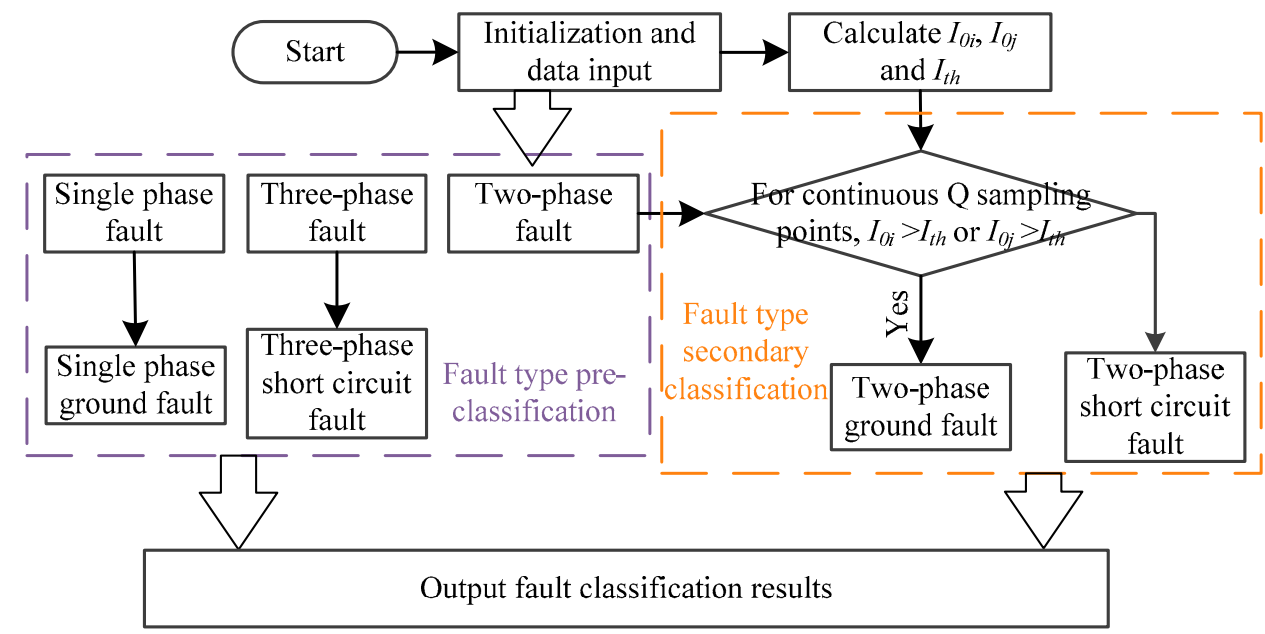

Figure 6. Flow chart of fault classification.

The zero-sequence current threshold setting equation is designed as follows:

$$
I_{t h}=\eta_{x} I_{z} .
$$

Therein, $I_{t h}$ is the threshold; $I_{z}$ is 10 percent of the maximum effective value of phase current; $\eta_{x}$ is the security coefficient, generally set as 1.0 3.0.

The procedures of fault classification method are illustrated as follows:

(1) Initialization and data input. Input $Q$, node three-phase currents, the sliding window parameters, and the fault detection result.

(2) Calculation. Calculate the effective value of zero-sequence current for both upstream node $\left(I_{0 i}\right)$ and downstream node $\left(I_{0 j}\right)$. Calculate the threshold with Equation (12).

(3) Fault type pre-classification. Input the fault detection result obtained in Section 3.2. If the fault is single phase fault, the fault type is single phase-to-ground fault. Enter into step 5. If the fault is three-phase fault, the fault type is three-phase fault. Enter into step 5. If the fault is two-phase fault, enter into step 4.

(4) Fault type secondary identification. If the effective value of zero-sequence is bigger than the threshold for continuous $Q$ sampling points, the fault type is two phase-to-ground fault. Otherwise, the fault type is two phase-to-phase fault.

(5) Output. Output the specific fault information including the fault phase and the fault type.

(6) Enter into the next moment and repeat steps 1-6. 


\section{Discussion}

To test the proposed method, simulation is completed with MATLAB/Simulink. Different situations for both section and system have been simulated and discussed.

\subsection{Section Simulation}

\subsubsection{Simulation of Fault Conditions}

A simulation is made for sections of double-ended power supply type and sections of power-load type. The time consumed for fault detection and fault classification is recorded to evaluate the detection time. The simulation model is shown in Figure 2. The fault ground resistance is set as $0.01 \Omega$. The fault happens at $0.8 \mathrm{~s}$. The sampling frequency of the current signal is $5 \mathrm{kHz}$. Ten types of fault (A phase-to-ground fault (Ag), B phase-to-ground fault (Bg), C phase-to-ground fault (Cg), A-B phase-to-ground fault (ABg), B-C phase-to-ground fault (BCg), A-C phase-to-ground fault (ACg), A-B phase-to-phase fault (AB), B-C phaseto-phase fault (BC), A-C phase-to-phase fault (AC), three-phase fault (ABC)) and different fault locations are considered. The simulation results are shown in Figures 7 and 8.

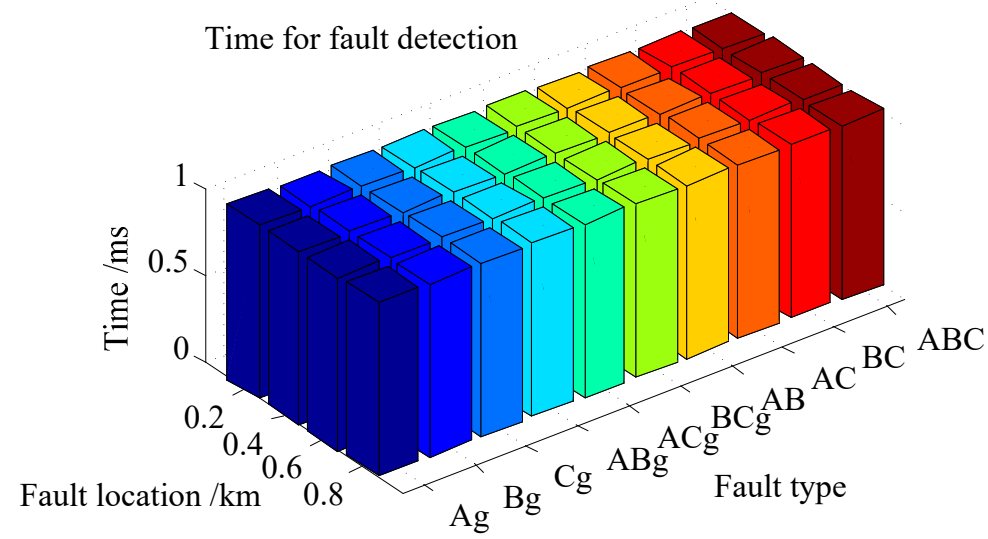

(a) Time consumed for fault detection.

Time for fault classification

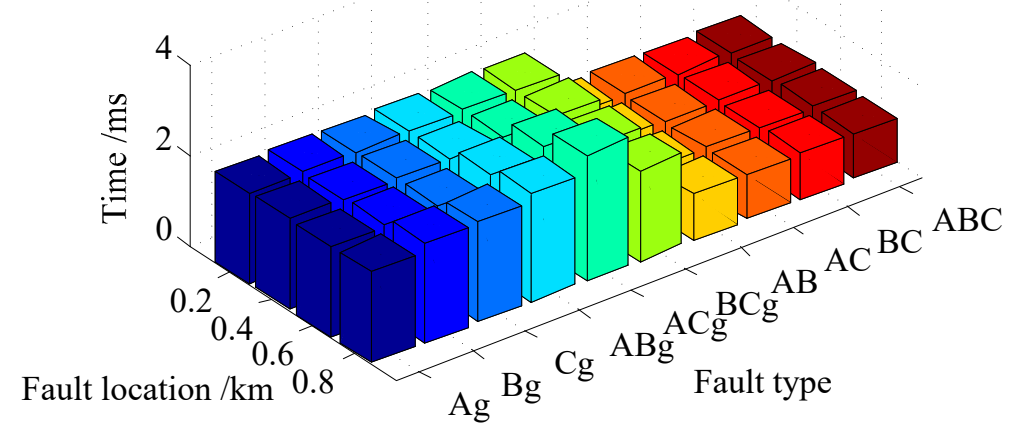

(b) Time consumed for fault classification.

Figure 7. Simulation result of the double-ended power supply section. 


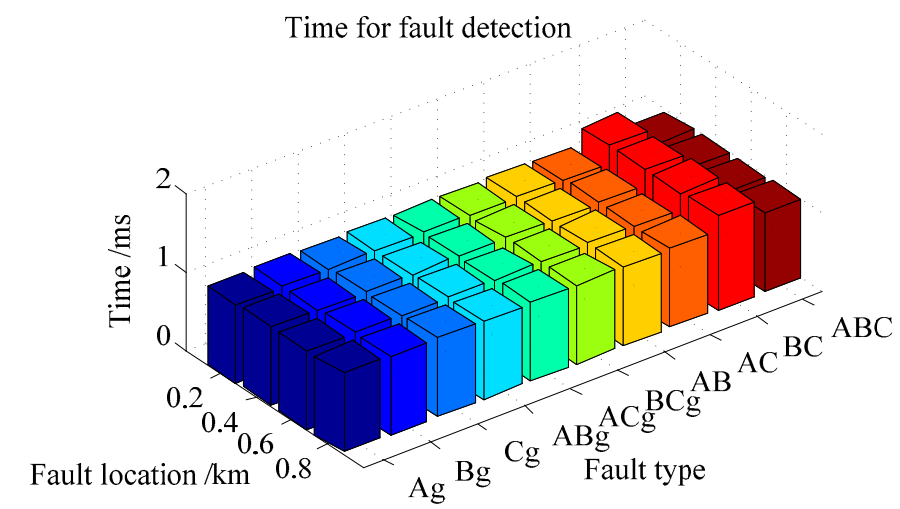

(a) Time consumed for fault detection.

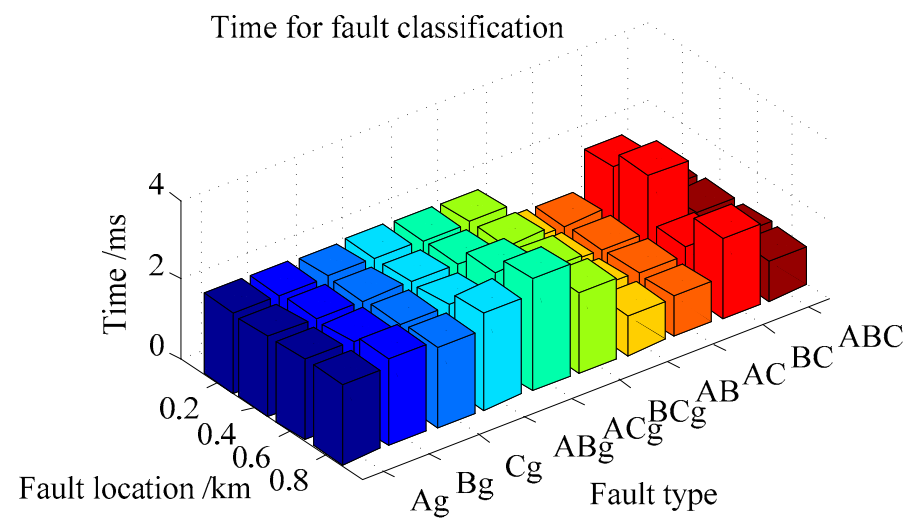

(b) Time consumed for fault classification.

Figure 8. Simulation result of the power-load section.

Under all simulation circumstances, the fault is detected and identified correctly. For all the simulation examples, the maximum fault detection time consumed is $1.2 \mathrm{~ms}$. The maximum fault classification time is $2.8 \mathrm{~ms}$. The results illustrate that the proposed method can detect faults rapidly and identify fault type accurately.

The detection of single phase high-impedance ground fault is also considered. Different ground resistances are set for simulation analysis based on the simulation model shown in Figure 2. The time consumed for fault detection is shown in Table 1. The result indicates that the proposed method can accurately detect a single phase ground fault with a ground resistance no more than $1 \mathrm{k} \Omega$. However, the time consumed for fault detection increases with the rise in the ground resistance.

Table 1. High-impedance ground fault simulation result (ms).

\begin{tabular}{ccccccc}
\hline \multirow{2}{*}{ Impedance/ $\boldsymbol{\Omega}$} & \multicolumn{2}{c}{ Double-Ended Power Supply Type } & \multicolumn{3}{c}{ Power-Load Type } \\
\cline { 2 - 7 } & $\mathbf{A g}$ & $\mathbf{B g}$ & $\mathbf{C g}$ & $\mathbf{A g}$ & $\mathbf{B g}$ & $\mathbf{C g}$ \\
\hline 0.01 & 1.0 & 1.0 & 1.0 & 1.0 & 1.0 & 1.0 \\
1 & 1.0 & 1.0 & 1.0 & 1.0 & 1.0 & 1.0 \\
10 & 1.0 & 1.0 & 1.0 & 1.0 & 1.0 & 1.0 \\
100 & 2.2 & 2.6 & 4.4 & 3.4 & 1.8 & 28.6 \\
500 & 8.4 & 10.6 & 10.0 & 12.0 & 23.2 & 6.2 \\
1000 & 13.0 & 158.0 & 12.0 & 8.2 & 167.8 & 44.8 \\
\hline
\end{tabular}




\subsubsection{Simulation of Disturbance Situations}

Non-fault disturbance may weaken the effectiveness of a fault detection method. Thus, the simulation also considers the influence of disturbance, such as the connection of distributed generation, the cutting off of load, and the frequency fluctuation. The disturbance simulation model is shown in Figure 9. The disturbance simulation parameters are listed in Table 2. The range of frequency fluctuation is $50 \pm 0.5 \mathrm{~Hz}$.

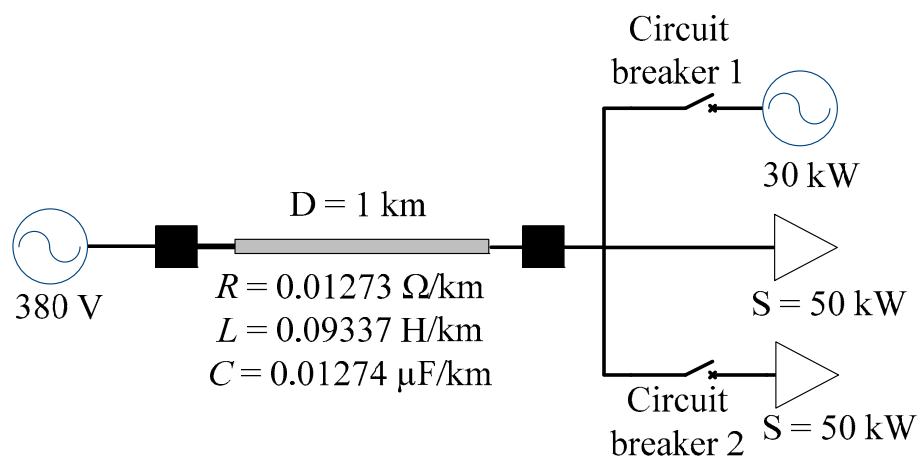

Figure 9. Disturbance simulation model.

Table 2. Disturbance simulation settings.

\begin{tabular}{ccc}
\hline Time/s & Load/\% & DG \\
\hline 0.6 & 100 & On \\
0.65 & 100 & Off \\
0.7 & 100 & On \\
0.8 & 200 & On \\
0.9 & 100 & On \\
\hline
\end{tabular}

The simulation results are shown in Figure 10. The blue line represents the IPDCHC; the black line represents the corresponding threshold; the red line represents the state of trip signal. As seen from Figure 10, under the three disturbance simulation situations, the IPDCHC remains lower than the threshold and the switch signal remains at low level. This indicates that no misjudgment happens, and the proposed method has a good anti-interference capacity.
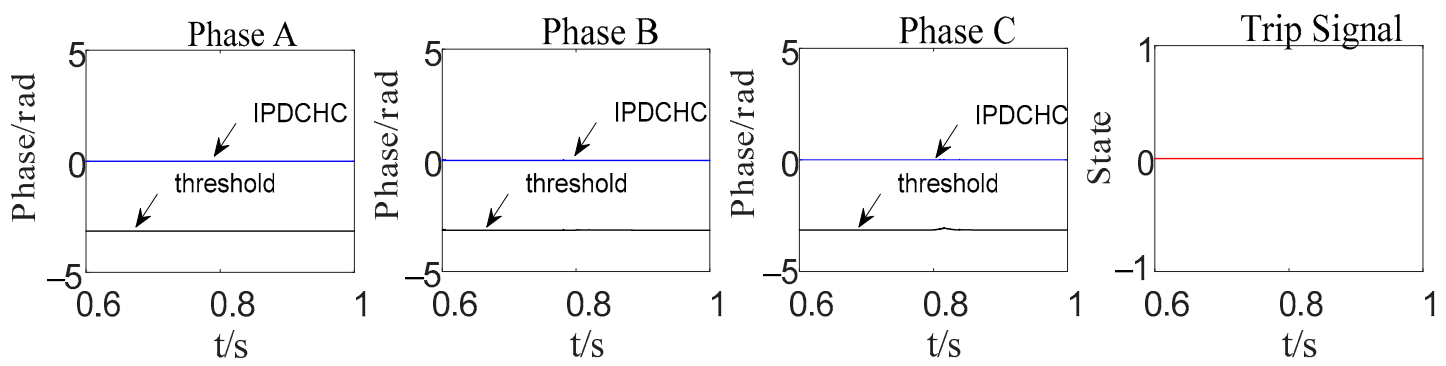

Figure 10. Simulation result of non-fault disturbance situation.

\subsection{System Simulation}

A microgrid system has two operation modes: grid-connected operation and island operation. The system model is displayed in Figure 11. Faults have been set in each section and 10 types of fault are all simulated. The fault ground resistance is $0.01 \Omega$ and the fault happens at $0.8 \mathrm{~s}$. The sampling frequency is $5 \mathrm{kHz}$. The fault locations of each section are listed in Table 3. 


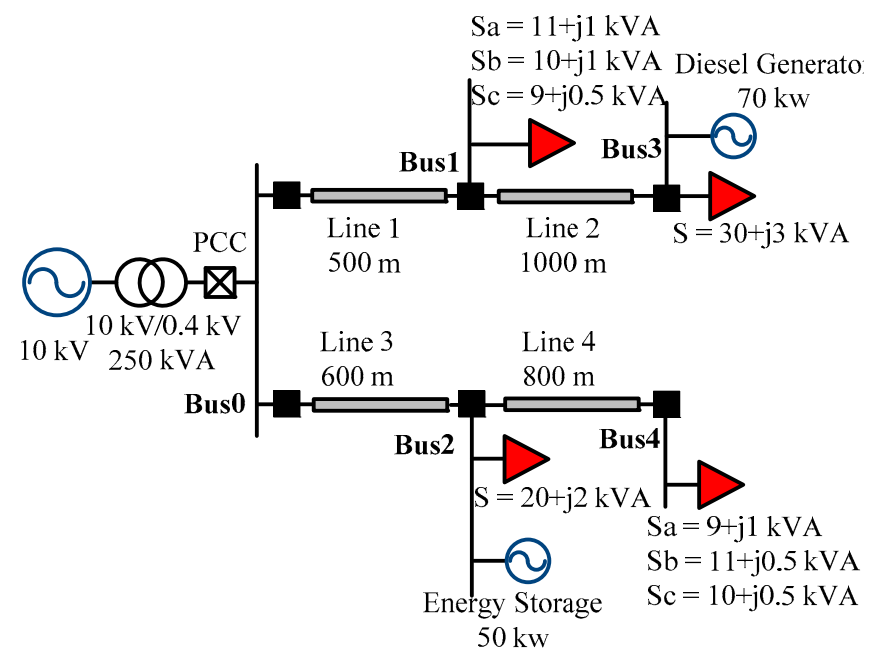

Figure 11. System simulation model.

Table 3. Fault location settings.

\begin{tabular}{ccccc}
\hline Section & L1 & L2 & L3 & L4 \\
\hline \multirow{2}{*}{ Fault location } & From BUS0 & From BUS1 & From BUS0 & From BUS2 \\
& $0.2 \mathrm{~km}$ & $0.7 \mathrm{~km}$ & $0.4 \mathrm{~km}$ & $0.3 \mathrm{~km}$ \\
\hline
\end{tabular}

The simulation results are shown in Figure 12. Under grid-connected operation mode, the maximum fault detection time is $13.8 \mathrm{~ms}$ and the maximum fault type identification time is $14.8 \mathrm{~ms}$. Under island operation mode, the maximum fault detection time is $4.8 \mathrm{~ms}$ and the maximum fault type identification time is $5.8 \mathrm{~ms}$. This illustrates that the proposed method can rapidly and accurately achieve fault detection and fault classification in a microgrid.

\section{Time for fault detection}

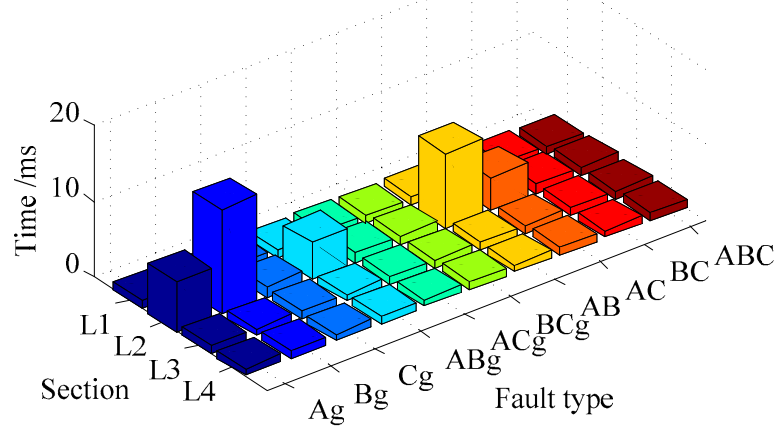

(a) Time consumed for fault detection under grid-connected operation mode.

Figure 12. Cont. 
Time for fault classification

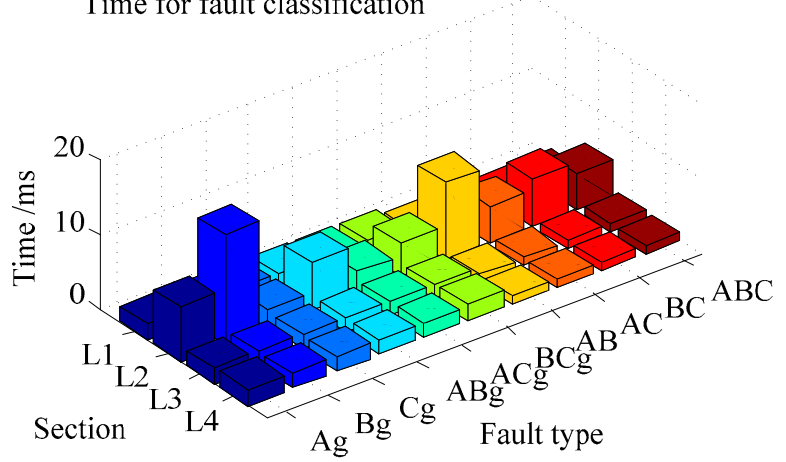

(b) Time consumed for fault classification under grid-connected operation mode.

Time for fault detection

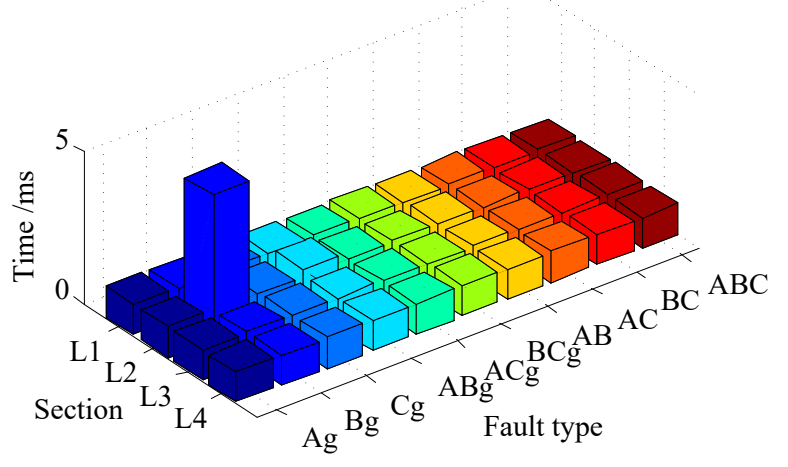

(c) Time consumed for fault detection under island operation mode.

Time for fault classification

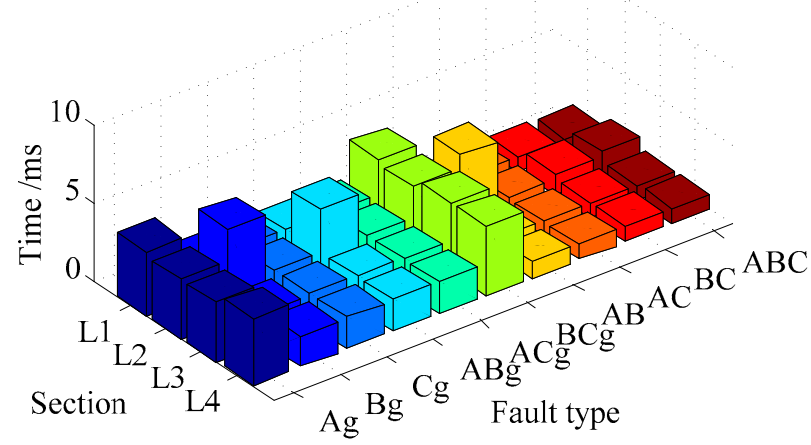

(d) Time consumed for fault classification under island operation mode.

Figure 12. Fault simulation results of a microgrid system.

\section{Conclusions}

A rapid and self-adaptive fault detection and classification method for microgrids is proposed in this paper. The instantaneous phase difference of the current high-frequency component (IPDCHC) extracted by HHT is used as the fault characteristic. The fault detection is made by comparing the IPDCHC and the self-adaptive threshold. The effective value of zero-sequence current is used to further identify the fault type. Thus, the complete fault information can be obtained. Section simulations and system simulations have been completed to test the proposed method.

The simulation results illustrate that the IPDCHC is an effective fault characteristic for detecting ten kinds of faults. Due to the utilization of IMF1 and the self-adaptive threshold setting method, fault detection can be carried out rapidly and accurately. The maximum fault detection time is $13.8 \mathrm{~ms}$ and the maximum fault type identification time is $14.8 \mathrm{~ms}$. The proposed method can also be applied to both grid-connected and island operation mode. No misjudgement happen under non-fault disturbance conditions, indicating that 
the method shows good self-adaptivity and anti-interference capacity. Moreover, the method can also detect single phase high-impedance ground faults. The maximum ground resistance is $1 \mathrm{k} \Omega$.

Author Contributions: Conceptualization, methodology, and writing, Y.L.; methodology and formal analysis, J.L.; supervision and writing review, G.N.; supervision, M.W.; investigation, X.W. All authors have read and agreed to the published version of the manuscript.

Funding: This research was funded by the National Natural Science Foundation of China, grant number 51877201, and the Cross-training program for high-level talents in colleges of Beijing, grant number 2019110750313.

Data Availability Statement: Not applicable.

Conflicts of Interest: The authors declare no conflict of interest.

\section{References}

1. Barra, P.H.A.; Coury, D.V.; Fernandes, R.A.S. A survey on adaptive protection of microgrids and distribution systems with distributed generators. Renew. Sust. Energ. Rev. 2020, 118, 109524-109539. [CrossRef]

2. Parhizi, S.; Khodaei, A.; Bahramirad, S. State of the art in research on microgrids: A review. IEEE Access 2015, 3, 890-925. [CrossRef]

3. Yu, J.Q.; Hou, Y.; Lam, Y.S.; Li, O.K. Intelligent Fault Detection Scheme for Microgrids With Wavelet-Based Deep Neural Networks. IEEE Trans. Smart Grid 2019, 10, 1694-1703. [CrossRef]

4. Hatziargyriou, N.; Asano, A.; Iravani, R.; Marnay, C. Microgirds. IEEE Power Energy Mag. 2007, 4, 78-94. [CrossRef]

5. Buraimoh, E.; Davidson, I.E.; Martinez-Rodrigo, F. Fault Ride-Through Enhancement of Grid Supporting Inverter-Based Microgrid Using Delayed Signal Cancellation Algorithm Secondary Control. Energies 2019, 12, 3994-4020. [CrossRef]

6. Maha, P.; Chen, Z.; Bak-Jensen, B.; Bak, C.L. A Simple Adaptive Overcurrent Protection of Distribution Systems with Distributed Generation. IEEE Trans. Smart Grid 2011, 2, 428-437.

7. Fahim, S.R.; Sarker, S.K.; Muyeen, S.M.; Sheikh, M.R.I.; Das, S.K. Microgrid Fault Detection and Classification: Machine Learning Based Approach, Comparison, and Reviews. Energies 2020, 13, 3460. [CrossRef]

8. Jin, D.G.; Choi, J.C.; Won, D.J.; Lee, H.J.; Chae, W.K.; Park, J.S. A Practical Protection Coordination Strategy Applied to Secondary and Facility Microgrids. Energies 2012, 5, 3248-3265. [CrossRef]

9. Nimpitiwan, N.; Thomas, G.; Ayyanar, R.; Suryanarayanan, S. Fault current contribution from synchronous machine and inverter based distributed generators. IEEE Trans. Power Deliv. 2007, 22, 634-641. [CrossRef]

10. Brahma, S.M.; Girgis, A.A. Development of adaptive protection scheme for distribution systems with high penetration of distributed generation. IEEE Trans. Power Deliv. 2004, 19, 56-63. [CrossRef]

11. Cagnanoa, A.; Tugliea, E.D.; Mancarellab, P. Microgrids: Overview and guidelines for practical implementations and operation. Appl. Energy 2020, 258, 114039-114056. [CrossRef]

12. Kulshrestha, A.; Mahela, O.P.; Gupta, M.K.; Gupta, N.; Patel, N.; Senjyu, T.; Danish, M.S.S.; Khosravy, M. A Hybrid Fault Recognition Algorithm Using Stockwell Transform and Wigner Distribution Function for Power System Network with Solar Energy Penetration. Energies 2020, 13, 3519. [CrossRef]

13. Najy, W.K.; Zeineldin, H.H.; Woon, W.L. Optimal protection coordination for microgrids with grid-connected and islanded capability. IEEE Trans. Ind. Electron. 2013, 60, 1668-1677. [CrossRef]

14. Yazdanpanahi, H.; Li, Y.W.; Xu, W. A new control strategy to mitigate the impact of inverter-based DGs on protection system. IEEE Trans. Smart Grid 2012, 3, 1427-1436. [CrossRef]

15. Sharaf, H.M.; Zeineldin, H.H.; El-Saadany, E. Protection Coordination for Microgrids With Grid-Connected and Islanded Capabilities Using Communication Assisted Dual Setting Directional Overcurrent Relays. IEEE Trans. Smart Grid 2018, 9, 143-151. [CrossRef]

16. Muda, H.; Jena, P. Superimposed Adaptive Sequence Current Based Microgrid Protection: A New Technique. IEEE Trans. Power Deliv. 2017, 32, 757-767. [CrossRef]

17. Gusha, T.; Bukharia, S.B.A.; Haidera, R.; Admasiea, S.; Ohb, Y.; Choa, G.; Kima, C. Fault detection and location in a microgrid using mathematical morphology and recursive least square methods. Electr. Power Syst. Res. 2018, 102, 324-331. [CrossRef]

18. Manditereza, P.T.; Bansal, R.C. Fault detection and location algorithm for DG-integrated distribution systems. J. Eng. 2018, 15, 1286-1290. [CrossRef]

19. Zhao, J.; Bian, R.; Zhang, X. A Micro-grid Fault Section Positioning Method Based On Fault Steady State Component. In Proceedings of the 2nd International Conference on Electrical, Control and Automation (ICECA2018), Shanghai, China, 10-11 November 2018.

20. Guo, Z.; Yao, J.; Tan, Z. Hilbert-Huang transform-based transient busbar protection algorithm. IET Gnger. Transm. Distrib. 2015, 14, 2032-2039. [CrossRef]

21. Fahey, T.S.; Burbure, N.V. Single-phase tripping. IEEE Power Energy Mag. 2008, 6, 46-52. [CrossRef] 
22. Hooshyar, A.; El-Saadany, E.F.; Sanaye-Pasand, M. Fault type classification in microgrids including photovoltaic DGs. IEEE Trans. Smart Grid 2016, 7, 2218-2229. [CrossRef]

23. Abdelgayed, T.; Morsi, W.; Sidhu, T. A New Approach for Fault Classification in Microgrids Using Optimal Wavelet Functions Matching Pursuit. IEEE Trans. Smart Grid 2017, 9, 4838-4846. [CrossRef]

24. Baloch, S.; Muhammad, M.S. An Intelligent Data Mining-Based Fault Detection and Classification Strategy for Microgrid. IEEE Access 2021, 9, 22470-22479. [CrossRef]

25. Huang, N.E.; Shen, Z.; Long, S.R.; Wu, M.C.; Shih, H.H.; Zheng, Q.; Yen, N.C.; Tung, C.C.; Liu, H.H. The empirical mode decomposition and the Hilbert spectrum for non linear and non-stationary time series analysis. Proc. R. Soc. Lond. A 1998, 454, 903-995. [CrossRef]

26. Yan, R.; Gao, R.X. Hilbert-Huang Transform-Based Vibration Signal Analysis for Machine Health Monitoring. IEEE Trans. Instrum. Meas. 2006, 55, 2320-2329. [CrossRef]

27. Hemapriya, C.K.; Suganyadevi, M.V.; Krishnakumar, C. Detection and classification of multi-complex power quality events in a smart grid using Hilbert-Huang transform and support vector machine. Electr. Eng. 2020, 102, 1681-1706. [CrossRef]

28. Gururani, A.; Mohanty, S.R.; Mohanta, J.C. Microgrid protection using Hilbert-Huang transform based-differential scheme. IET Gener. Transm. Distrib. 2016, 10, 3707-3716. [CrossRef]

29. Chen, Y.M.; Zi, Y.Y.; Cao, H.R.; He, Z.J.; Sun, H.L. A data-driven threshold for wavelet sliding window denoising in mechanical fault detection. Sci. China Technol. Sci. 2014, 57, 589-597. [CrossRef] 